\title{
Alter
}

Revue de phénoménologie

$28 \mid 2020$

La religion

\section{Une lettre inédite de Husserl à Jean Héring, 4} février 1937

\section{OpenEdition}

Journals

Édition électronique

URL : https://journals.openedition.org/alter/2214

DOI : 10.4000/alter.2214

ISSN : 2558-7927

Éditeur :

Association ALTER, Archives Husserl (CNRS-UMR 8547)

Édition imprimée

Date de publication : 1 novembre 2020

Pagination : 307-315

ISBN : 978-2-9550449-6-4

ISSN : 1249-8947

Référence électronique

"Une lettre inédite de Husserl à Jean Héring, 4 février 1937 », Alter [En ligne], 28 | 2020, mis en ligne le 22 décembre 2020, consulté le 12 décembre 2021. URL : http://journals.openedition.org/alter/2214 ; DOI : https://doi.org/10.4000/alter.2214

Ce document a été généré automatiquement le 12 décembre 2021.

Revue Alter 


\section{Une lettre inédite de Husserl à Jean Héring, 4 février 1937}

1 Élève de Husserl à Göttingen entre 1910 et 1913, condisciple d'Edith Stein, Alexandre Koyré et Hedwig Conrad-Martius, avec qui il cultiva les liens d'une amitié durable, Jean Héring, né en 1890 en Alsace - alors territoire du Reich allemand -, a pris la nationalité française en 1918. Sa première contribution en phénoménologie, les Bemerkungen über das Wesen, die Wesenheit und die Idee, voit le jour en 1921 dans le Jahrbuch für Phänomenologie und phänomenologische Forschung édité par son maître. Après avoir poursuivi ses études à Paris, à l'École Pratique des Hautes Études, où il suit notamment les cours d'Étienne Gilson, Héring soutient une thèse en 1925 pour obtenir la licence de théologie, Phénoménologie et philosophie religieuse. Étude sur la théorie de la connaissance religieuse (Paris, Alcan, 1926), première monographie de langue française ouvertement inscrite dans le mouvement philosophique inauguré par Husserl. Héring est ainsi un acteur fondamental de la toute première réception de Husserl en France ${ }^{1}$, bien au-delà du fait d'avoir orienté le jeune Levinas vers Fribourg-en-Brisgau et d'avoir ainsi donné une impulsion décisive à sa thèse sur La théorie de l'intuition dans la phénoménologie de Husserl (1930), au jury de laquelle il siégera avant d'en donner la recension, deux ans plus tard, dans la Revue philosophique de la France et de l'étranger.

2 Auteur également d'un mémoire en sciences religieuses Sur la doctrine de la chute et de la préexistence des âmes chez Clément d'Alexandrie (EPHE, 1923), Héring intègre la Faculté de Théologie protestante de Strasbourg où, après sa thèse sur Le royaume de Dieu et sa venue. Étude sur l'espérance de Jésus et de l'apôtre Paul (1937), il occupera la chaire de Nouveau Testament jusqu'à sa retraite en 1956. Ses articles et les nombreuses recensions publiées dans la Revue d'histoire et de philosophie religieuse ${ }^{2}$ témoignent du fait qu'il a continué, pendant tout ce temps, à s'intéresser activement au mouvement phénoménologique. Par ailleurs, Héring a entretenu des relations amicales et fidèles avec les époux Husserl, leur rendant visite régulièrement, et a organisé à Strasbourg, dans un climat et des circonstances très différentes, deux des conférences de son maître, en 1929 et en 1937.

3 La Correspondance de Husserl et Héring éditée par Karl Schuhmann en 1994 dans la collection Husserliana Dokumente (vol. III/3) se réduit à une poignée de lettres, dont fait 
partie notamment l'échange autour de la question du rêve traduit par Philippe Ducat en 1997 dans le numéro 5 d'Alter. Identifiée par Édouard Mehl en 2020, la lettre que nous éditons et traduisons ici vient, entre autres documents, enrichir le dossier de la correspondance de Husserl, et témoigne de ses liens pérennes avec un ancien disciple devenu - comme le phénoménologue fribourgeois le décrit souvent à ses interlocuteurs dans sa Correspondance - un « vieil ami».

4 Après la mort de Héring (1966), ses papiers personnels ont été déposés par l'Université à la bibliothèque $\mathrm{du}$ "Stift» (Collegium Wilhelmitanum, Fondation St-Guillaume Médiathèque protestante de Strasbourg). Un premier classement de ces papiers a été fait par Gustave Koch, puis un inventaire des papiers classés a été établi par le Révérend Joachim Feldes ${ }^{3}$. La lettre que nous publions ici ne figurait pas dans la première version de cet inventaire : un doute (peu fondé par ailleurs) sur l'identité de son destinataire fait qu'elle avait été rejetée et placée, en attente d'authentification définitive, dans un carton de documents non classés. Sa lecture apporte une confirmation supplémentaire des rapports privilégiés que Husserl a gardés avec Héring tout en laissant transparaître ses sentiments chaleureux pour le milieu académique strasbourgeois. Il faut en effet rappeler que l'année 1937 est celle du Congrès Descartes de Paris, auquel Husserl se vit refuser l'autorisation de participer. Dans ce contexte, les échanges - encore possibles avec Strasbourg ont pu recevoir à ses yeux une importance et une valeur particulières.

Le choix d'éditer cette lettre dans la revue de phénoménologie ALTER en annexe d'un dossier thématique sur la religion a été dicté par la manière dont Husserl y avoue son appétence pour des questions théologiques, suggérant que celles-ci entrent en résonance étroite avec la signification qu'il attribue à ses derniers travaux et à son œuvre tout entière. Ainsi, lorsqu'il exprime son vif intérêt pour les travaux des collègues théologiens de Héring, Husserl affirme l'« orientation théologique qui lui est propre » - bien qu'il reconnaisse n'être aucunement théologien au sens disciplinaire, académique ou strict du terme -, et va jusqu'à souligner la signification «théologique » (entre guillemets, donc toujours en un sens qui lui est propre) de son dernier ouvrage, La crise des sciences européennes et la phénoménologie transcendantale (1936).

L'établissement du texte est l'œuvre de Thomas Vongehr (Archives Husserl Louvain) ; la traduction, les annotations et la présentation sont dues à Édouard Mehl (Centre de recherches en philosophie allemande contemporaine, Strasbourg) et Claudia Serban (Équipe de recherche sur les rationalités philosophiques et les savoirs, Toulouse et Archives Husserl, Paris).

7 Freiburg i/B. 4. II 1937

8 Lieber Freund!

9 Das hohe Alter hat seine argen Beschwerden und gar in einer Zeit, die so eifrig beflissen scheint die ohnehin dem Verfall preisgegebenen Brücken zur Ewigkeit abzubrechen Autostrassen, Flugzeuge, Kanonen sind ihr wichtiger! Und doch, unverhofft strahlt in das Dunkel solchen Alters gelegentlich ein freudenweckendes Licht ein, die drohende Erstarrung löst sich, der Herzschlag wird lebendiger, neue Kraftströme verbreitend.

Eine solche beglückende Umstimmung verdanke ich (inmitten einer Periode seelischer Ermattung) der theologischen Gabe, die Sie mir, l<ieber> Freund zugehen liessen. Welch schönes Zeugnis für Ihren dahin gegangenen edlen Senior Baldensperger, dem sie als Festschrift zugedacht war, und zugleich für den reinen und hohen Geist, der in Ihrer ganzen Fakultät waltet! Hoch erfreut es mich, dass Sie mir diese Festschrift im Namen 
ihrer Verfasser überreichen durften. Ich kann Ihnen, Ihnen allen dafür nicht genug danken.

11 Ich habe mich sogleich in den Abhandlungen umgethan $u$. schon im ersten Umblicken erfuhr ich in mir starke Resonanzen - Resonanzen welche die behandelten theologischen Probleme und ihre Ausführungen in mir, dem ganz Untheologischen und doch in einem eigenen Sinn ganz theologisch gerichteten erregen - in mir, der soeben das erste Stück einer Schrift publiziert hat, deren Titel und erster Gang freilich nicht erwarten lässt, dass sie ihrem letztlichen Sinn nach „theologisch“ ist. Mindestens die Untersuchungen der Herrn Hauter, Ménégoz, Monod (vermutlich auch Strohl und Will) geben mir zu denken, „sprechen mich“ - den Phänomenologen - „an“. Das bisher vorliegende Stück meiner Schrift (seit 1 Monat warte ich auf die Separata!) wird was ich als „theologisch“ meine, wie gesagt, nochnicht verständlich bekunden, zunächst nicht: wie sehr mein neuer "transzendental “ betitelter Weg einer universalen phänomenologischen Seinsbetrachtung darauf aus ist die Wesenscorrelation zwischen der historischen (überhaupt der concreten) Facticität und der sich darin, durch objektivierung, durch Veräusserlichung, verhüllenden absoluten Geistigkeit zur Aufweisung zu bringen. Darin liegt aber zugleich: zu einer in einem völlig neuen Sinne streng wissenschaftlichen Erkenntnis der universalen absoluten Teleologie und der wissenschaftlich wirklich fassbaren Probleme der Offenbarung des in sich absolut einigen Absoluten - Offenbarung in einem weiten Sinn, darunter der religiösen offenbarung.

12 Es treten also als transzendental-phänomenologische Probleme und wirklich als wissenschaftliche „Gott", Unsterblichkeit (diese im irdischen Leben, irdischer Geburt $u$. ird<ischen> Tod verhüllt) $u$. teleologisches Weltverständnis als einzig denkbares etc. auf - aber in einem Sinn u. einer Wissenschaftlichkeit, die im äussersten Contrast zur scholastischen und selbst der Kant- transzendentalen steht. Doch wie weit mir noch die literarische Ausgestaltung der ganzen Schrift vergönnt ist - mir der im $8^{\text {ten }}$ Lebensjahrzehnt schon so hoch auf- oder viel mehr abgestiegen ist - mir gebunden an die phänomenologische, also ernstlich aufweisende Methode, die alles argumentierende, alles hypothetisch substruierende und logisch, ob inductiv oder deductiv beweisende Verfahren als in der Weltlichkeit verhaftet perhorresziert, das obliegt der göttli<chen> Gnade. Aber ich, so wie ich bin, möchte doch ruhig sterben können - lebensmüde bin ich wahrhaftig genug - und das kann ich nicht ohne meinen Auftrag von oben - als wie ich ihn erlebe - vollendet zu haben: also ohne den Anfang einer absoluten, das Absolute, den Sinn des Daseins wirklich erreichenden Philosophie paradox gesprochen vollendet $\mathrm{zu}$ haben. Also als Ergebnis meines Lebens die wohlbegründete „Landkarte“ des gelobten Landes der echten Erkenntnisse, in totalen sicheren Umrissen - dazwischen die weissen Flecken, als Anzeigen der künftigen Entdeckungen ins Unendliche. Und hier resultiert die allein radicale und endgiltig wirksame Widerlegung aller Skeptizismen und Negativismen. Sie alle ziehen ihre Verführungskraft aus der positiven Wissenschaft und aus der Blindheit dafür dass solche Wissenschaftlichkeit nur Techné ist und dass einzig eine Wissenschaft aus absolutem Grunde, also eine transz<endental> teleologische, ernstlich „erklären“ kann, ernstlich Verständnis, Einsicht schaffen. Unphilosophische Naivität des Lebens, mit seinen mannigfaltigen Wahrheiten (Sachwahrheiten, ethischen, religiösen etc.) und den entsprechend zugehörigen naiven Evidenzen hat ihr nie, und durch keinen Skeptizism, zu verkümmerndes Recht. Aber die Wissenschaft der Jahrtausende vermochte dieses Recht nicht $\mathrm{zu}$ wahren, eben vermöge ihrer bloss technisch 
fruchtbaren, aber für das Absolute, des für jederlei Sein $u$. Wahrheit sinngebende, blinden Positivität - der Mutter aller Skepsis. Diese aber vergiftete schliesslich das gesamte Menschheitsleben u. schuf jene, in allem und selbst im Geistigen äusserliche, nur technisch fortschreitende Cultur, der aller seelenerbauende Sinn fehlte. Im Bewusstsein dieses Sinnes, des göttlichen Sinnes, weiss sich der Mensch und die vergemeinschaftete Menschheit, in ihrer Endlichkeit doch als Funktionär des Absoluten, das in ihr, in Jedem, so weit sich offenbart, activ schöpferisch waltend, als er freier ist, frei den Auftrag von oben erfüllend.

Doch verzeihen Sie, dass ich mich philosophisch so weit treiben liess - senectus loquax! Aber Sie sehen daraus, wie tief mich das bedeutsame Geschenk dieser Festschrift angeregt und erfreut hat.

Ich verstehe lieber Freund, dass Sie sich, was Sie so oft mir sagten, in diesem Kreise so glücklich $u$. als Theologe so glücklich fühlen. So muss ich zufrieden sein, wie sehr ich dereinst es bedauerte, dass ich auf Ihre phänomenologische Mitarbeit verzichten musste.

Mit wiederholtem herzlichen Dank und freundlichen Grüssen an die Herren Collegen

La vieillesse nous réserve d'âpres tourments, surtout en cette époque qui semble si fort attachée à briser les ponts vers l'éternité, qui ont de toute façon commencé à se délabrer - les autoroutes, les avions et les canons lui importent davantage ! Et pourtant, dans les ténèbres d'un tel âge brille de temps à autre, de manière inespérée, une lumière éveillant la joie: la paralysie menaçante se dissipe, le cœur reprend de la vigueur, dégageant de nouveaux effluves de force.

21 En proie à ce découragement spirituel, je suis redevable d'un si heureux retournement d'humeur à l'offrande théologique que vous m'avez permis, cher ami, de découvrir. Quel beau témoignage pour votre noble aîné récemment disparu, M. Baldensperger ${ }^{4}$, auquel était destiné cet hommage, ainsi que pour l'esprit pur et élevé qui règne dans toute votre Faculté! Je me réjouis vivement que vous m'ayez fait parvenir ce volume d'hommage au nom de son auteur. Je ne saurais vous en remercier - vous tous - assez !

Je me suis plongé aussitôt dans ces études ${ }^{5}$ et déjà aux premiers aperçus j'ai ressenti de fortes résonances - des résonances que les problèmes théologiques abordés et leurs traitements suscitent en moi, qui ne suis pas du tout théologien et qui pourtant, en un sens qui m'est propre, suis orienté de manière entièrement théologique; en moi qui viens de publier la première partie d'un ouvrage ${ }^{6}$ dont le titre et la démarche initiale ne laissent certes pas présager qu'il est, en son sens ultime, «théologique». Les recherches de Messieurs Hauter, Ménégoz, Monod (sans doute aussi Strohl et Will) me donnent au moins à penser, « m'interpellent »- moi, le phénoménologue ${ }^{7}$. La partie de mon ouvrage qui est à présent disponible (j’attends mes exemplaires depuis un mois !) ne manifestera pas encore de manière compréhensible ce que j'entends par "théologique ", comme je viens de le dire ; elle ne montre pas d'emblée combien mon nouveau chemin dit «transcendantal» - celui d'une considération phénoménologique universelle de l'être - a à charge de manifester la corrélation d'essence entre la facticité historique (ou plus généralement concrète) et la spiritualité absolue qui s'y dévoile en 
s'objectivant et en s'extériorisant. Or cela implique également ceci : [il faut porter cette corrélation] à une connaissance, rigoureusement scientifique en un sens entièrement nouveau, de la téléologie absolue universelle et des problèmes, effectivement appréhendables de manière scientifique, de la révélation de l'Absolu qui est absolument unitaire en soi - révélation au sens le plus large, qui comprend la Révélation au sens religieux.

Entrent donc en compte, en tant que problèmes de phénoménologie transcendantale et effectivement scientifiques, "Dieu», l'immortalité (celle-ci étant voilée dans la vie terrestre, dans la naissance terrestre et la mort terr<estre>) et la compréhension téléologique qui permet de penser le monde dans son unicité, etc. - mais en un sens et avec une dimension scientifique qui contraste de manière extrême avec leur signification scolastique, et même avec celle que leur confère le transcendantalisme kantien. Pourtant, savoir jusqu'où il me sera donné d'aller dans la mise en forme de l'ouvrage entier - à moi qui suis déjà monté si haut ou plutôt descendu dans ma huitième décennie de vie-,moi qui suis lié à la méthode phénoménologique rigoureusement démonstrative, abhorrant toute démarche ratiocinante qui procède par substructions hypothétiques ou se contente de preuves logiques inductives ou déductives et qui reste ancrée dans la mondanéité, cela relève de la grâce divine. Mais tel que je suis, je voudrais pouvoir mourir en paix - car je suis vraiment assez fatigué de vivre - et cela, je ne le peux pas tant que je n'ai pas achevé ma mission d'en haut (c'est ainsi que je la vis) : donc sans avoir mené à terme le commencement (pour le dire d'une manière paradoxale) d'une philosophie absolue, qui atteint effectivement l'absolu et le sens de l'existence. Le résultat de ma vie [serait] ainsi la «carte» de la Terre promise de la connaissance véritable, avec des contours entièrement assurés - et, entre eux, les taches blanches comme indices de découvertes futures [se poursuivant] à l'infini. Et cela fournit la seule réfutation de tous les scepticismes et négativismes qui soit radicale et efficace de manière définitive. Ceux-ci tirent tous leur force de séduction de la science positive, ainsi que de la cécité pour le fait qu'une telle scientificité n'est que techné et que seule une science élaborée à partir de fondements absolus, donc une science téléologique transc<endantale>, peut "expliquer » sérieusement et produire véritablement une vue évidente et une compréhension. La naïveté non philosophique de la vie, avec ses diverses vérités (vérités de fait, éthiques, religieuses, etc.) et les évidences naïves correspondantes qui lui appartiennent n'a aucunement le droit d'altérer [une telle science] par un quelconque scepticisme. Mais la science du siècle n'a pu respecter ce droit, précisément à cause de sa positivité qui n'était féconde que du point de vue technique ; elle était ce faisant aveugle à l'absolu qui donne être et vérité à tout - cette positivité qui est la mère de toute attitude sceptique. Celle-ci a empoisonné au bout du compte toute la vie de l'humanité et a forgé une culture en tout point extérieure - même d'un point de vue spirituel -, une culture qui ne progressait que de manière technique et à laquelle manquait tout sens spirituellement édifiant. Ayant conscience de ce sens, ce sens divin, l'être humain et l'humanité communautarisée a pourtant, dans sa finitude même, le savoir d'être le fonctionnaire de l'absolu, qui se révèle en lui et en chacun et y règne d'une manière activement créatrice d'autant mieux qu'il est plus libre - libre pour autant qu'il remplit sa mission d'en haut.

Veuillez me pardonner si je me suis laissé emporter aussi loin d'un point de vue philosophique - senectus loquac[ior]! Mais vous pouvez voir ainsi combien profondément le présent remarquable de ce volume d'hommage m'a intéressé et réjoui. 
Je comprends, cher ami, que, comme vous me l'avez dit si souvent, vous vous sentiez si heureux, et si heureux en tant que théologien, au sein de ce cercle. Je dois ainsi être content, bien que cela m'ait beaucoup attristé à un moment, d'avoir dû renoncer à votre collaboration en tant que phénoménologue ${ }^{8}$.

Messieurs vos collègues,

Votre vieil ami,

EHusserl

\section{NOTES}

1. Nous renvoyons à ce propos aux recherches récentes de C. Dupont dans Phenomenology in French Philosophy: Early Encounters, Dordrecht, Springer, 2014 et «Jean Héring and the Introduction of Husserl's Phenomenology to France ", Studia Phønomenologica, vol. XV (2015), p. 129-153. Voir également N. Monseu, Les usages de l'intentionnalité. Recherches sur le première réception de Husserl en France, Peeters, Louvain-La-Neuve, 2005, les travaux réunis dans Jean Héring: De l'eidétique à la phénoménologie de la religion, dossier coordonné par C. Serban et D. Pradelle, Revue de théologie et de philosophie, vol. 148, n 1/2016, ainsi que la contribution d'É. Mehl, «La "philosophie religieuse" de Jean Héring", Revue d'histoire et de philosophie religieuse, à paraître.

2. Dans deux numéros d'hommage qui lui ont été consacrés, en 1957 et 1966, la Revue d'histoire et de philosophie religieuse a recensé les principales publications de Héring. Ces bibliographies sont disponibles ici : https://www.persee.fr/doc/rhpr_0035-2403_1957_num_37_1_3481 et https:// www.persee.fr/doc/ rhpr_0035-2403_1966_num_46_2_3829\#rhpr_0035-2403_1966_num_46_2_T1_0113_0000.

3. Les Archives Jean Héring, créées en 2020, se donnent pour mission de compléter cet inventaire et d'entreprendre la publication d'une correspondance en grande partie inédite.

4. Rigoureusement contemporain de Husserl, Guillaume Baldensperger (1856-1936) a étudié à Strasbourg, à Göttingen sous la direction d'Albrecht Ritschl (dont Héring récuse le subjectivisme outré dans Phénoménologie et philosophie religieuse) et à Paris. Ordonné pasteur en 1880, Baldensperger a été professeur de théologie et directeur du séminaire à Giessen (1892-1915), professeur à Lausanne de 1916 à 1919, pour rejoindre enfin la Faculté de Théologie protestante de l'Université de Strasbourg à partir de 1919 et y occuper, jusqu'à son éméritat en 1929, la chaire de Nouveau Testament que reprendra Héring après sa thèse de 1937. Dans son abondante bibliographie, plusieurs ouvrages ont jeté un éclairage nouveau sur l'étude du rapport entre eschatologie juive et chrétienne, à commencer par son étude pionnière Das Selbstbewusstsein Jesu im Lichte der messianischen Hoffnungen seiner Zeit (1888), ou - paru au moment où Baldenspeger rejoint l'Université française de Strasbourg - «L'apologétique de la primitive Église. Son influence sur la tradition des origines et du ministère galiléen de Jésus », Revue de théologie et de philosophie 8/34, 1920, p. 5-43. Sur Baldensperger comme les autres théologiens strasbourgeois cités dans cette lettre, voir Matthieu Arnold, La Faculté de Théologie Protestante de l'Université de Strasbourg de 1919 à 1945, Strasbourg, Association des Publications de la Faculté de Théologie protestante, 1990 (sur Baldensperger, p. 54-58). 
5. Le volume reçu par Husserl, et qui est conservé dans sa bibliothèque, constitue le premier numéro des Recherches théologiques. Par les professeurs de la Faculté de Théologie Protestante de l'Université de Strasbourg, Paris, Félix Alcan, 1936 - À la mémoire de Guillaume Baldensperger (1856-1936). Comme l'indique l'Avant-Propos, ce volume reproduit tel quel les $n^{\circ}$ 3-5 de la Revue d'histoire et de philosophie religieuses, vol. 16, mai-octobre 1936. Ce Cahier est disponible en ligne sur https://www.persee.fr/doc/rhpr_0035-2403_1936_num_16_3_2965. Il comprend les contributions suivantes: Jean Monnier, «Sur la grâce, à propos de la parabole de la brebis perdue »; Jean Héring, « Kyrios Anthropos »; Oscar Cullmann, «Le caractère eschatologique du

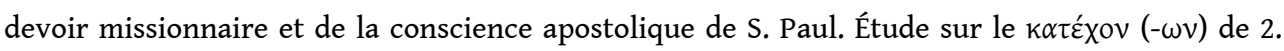
Thess. 2: 6-7»; Charles Jaeger, «Remarques philologiques sur quelques passages des Synoptiques »; William Seston, "L'opinion païenne et la conversion de Constantin »; Henri Strohl, «La notion d'Église chez les Réformateurs »; Charles Hauter, « La présence divine comme problème de la dogmatique protestante »; Fernand Ménégoz, «Résurrection »; Victor Monod, «Le voyage, le déracinement de l'individu hors du milieu natal constituent-ils un des éléments déterminants de la conversion religieuse ?»; Robert Will, « Le symbolisme de l'image du Christ. Essai d'iconographie chrétienne » et Théodore Gérold, «Les drames liturgiques médiévaux en Catalogne $»$.

6. Il s'agit de la Crise des sciences européennes, dont les deux premières sections (§§ 1-27) sont parues en 1936 à Belgrade, dans la revue Philosophia.

7. Dans son article «Résurrection" (Recherches théologiques, p. $171=$ RHPR, p. 355), F. Ménégoz cite les quelques lignes du Nachlass d'Adolf Reinach mises par Héring en tête de Phénoménologie et philosophie religieuse (1926): "Qu'avant tout on laisse aux expériences religieuses leur sens propre! Même si ce sens place l'observateur devant des énigmes... ». Ménégoz insiste sur le caractère imperceptible pour l'œil charnel de la résurrection, qui relève selon lui d'une forme d'« objectivité interne » qui ne passe pas dans l'objectivité « externe », non sans insister sur le fait que la Révélation du Ressuscité suppose un «moi » qui en fasse l'expérience, en citant à

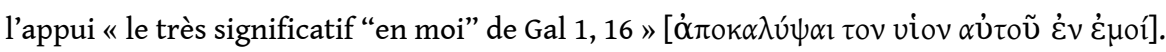

8. Curieusement, Husserl passe sous silence tout au long de sa lettre la contribution de Héring au volume d'hommage que celui-ci lui a envoyé, intitulée "Kyrios Anthropos ». Or, de toutes les contributions du numéro de 1936, cet article est celui qui est sans doute le plus intimement lié au travail de Baldensperger. En effet, la thèse qu'y soutient Héring - que Jésus ait revendiqué le titre de « Fils de l'Homme » (expression issue de l'apocalyptique juive et en particulier de Daniel), mais refusé celui de Messie, et que la messianité de Jésus soit donc une invention du christianisme primitif dans le cadre de l'évangélisation des Juifs - est très liée aux travaux de Baldensperger. Plus généralement, les études néotestamentaires de Baldensperger et de Héring conduisent à attribuer à l'humain - à la notion et au mot «Homme » - un sens eschatologique proprement irréductible à toutes les interprétations platement anthropologiques et subjectivistes (comme l'était celle de Ritschl, le maître de Baldensperger) de la Bible, et susceptible d'entrer en résonance avec la compréhension transcendantale et téléologique de l'humanité que propose Husserl. 